\title{
Accentuated eccentric training: effects on horizontal jump distance and muscle strength among young adults
}

\begin{abstract}
Accentuated eccentric loading is a training method in which greater load is applied during eccentric phase in comparison to concentric phase of a coupled eccentric-concentric action. The aim of this study was to evaluate the training effects of accentuated eccentric load countermovement jump on horizontal jump distance and muscle strength of lower extremity among young adults. This study was designed as a randomized controlled trial with pretest and post-test model. The subjects were randomly categorized into a control group $(n=15)$ that underwent body-mass countermovement jump and an intervention group $(n=15)$ submitted to Accentuated Eccentric Training. 30 subjects including 16 males and 14 females participated in the four-week long training. Accentuated Eccentric Training group showed significant improvement in horizontal jump distance and maximum muscle strength of lower extremities. An increase in horizontal jump distance $(+13.15 \%)$ and 5RM squat strength was noted $(+23.14 \%)$ after four weeks of training. In conclusion, it would appear that Accentuated Eccentric Training enhance athletic performance in young adults. Application of accentuated eccentric loading could be further investigated for its effects on agility and exercise induced muscle damage in athletes of different sports.
\end{abstract}

Volume 3 Issue 3 - 2018

\author{
Nizar Abdul Majeedkutty,' Pang Shi Yiing,' \\ Anila Paul ${ }^{2}$ \\ 'Department of Physiotherapy, University Tunku Abdul Rahman, \\ Malaysia \\ ${ }^{2}$ Department of Physiotherapy, Medical Trust College of \\ Physiotherapy, India
}

Correspondence: Nizar Abdul Majeedkutty, Department of Physiotherapy, University Tunku Abdul Rahman, Malaysia, Tel 0060163702375, Email nizarabdul@utar.edu.my

Received: May 04, 2018 | Published: June 12, 2018

Keywords: accentuated eccentric loading, countermovement jump, horizontal jump distance, maximum muscle strength

\section{Introduction}

Efficient and effective training methods are continually sought after by physiotherapists, coaches, and athletes to enhance sports performance, ${ }^{1}$ Resisted movement training is used in athletic conditioning to enhance power and athletic performance by executing a movement important to sport with added resistance that is not excessive and does not adversely affect the movement pattern. Traditional weight training exercises performed slowly with heavy resistance are well suited to enhance strength but may not be optimal for power development requiring higher velocity because there are considerable portions of deceleration during the motion, whereas resisted movement training can allow for acceleration throughout the range of motion. ${ }^{2}$ Eccentric exercises are frequently applied in strength and conditioning program by giving external resistance to the targeted muscles in the direction which is opposite the muscle pull. According to ${ }^{3}$ eccentric exercises improve maximal and explosive muscle strength, shift the optimal muscle length, improve muscle coordination and induce muscular adaptations. There are few different training modalities derived from eccentric exercises such as "pure eccentric" and "coupled eccentric-concentric" manner. Accentuated eccentric loading (AEL) is one of the extended concepts from eccentric exercises. Accentuated eccentric loading is a training method in which greater load is applied during eccentric phase in comparison to concentric phase of a coupled eccentric-concentric action. The aim of this study was to evaluate the training effects of accentuated eccentric load countermovement jump on horizontal jump distance and maximum muscle strength of lower extremity among young adults. According to ${ }^{4}$ AEL was described as magnitude of eccentric load is higher than that of concentric load while maintaining the natural mechanism of movements involving concentric and eccentric action. It means that an additional load is applied during eccentric phase in comparison to concentric phase of a movement. It is hypothesized that a greater concentric force will be developed through higher loading in eccentric action. It is believed that AEL involving coupled concentric and overloaded eccentric muscle actions could optimize the effects of stretch-shortening cycle (SSC). An additional mechanical stimulus was given during eccentric phase in order to produce greater force and power output during concentric phase in SSC activities. AEL augments the negative work of eccentric phase, thereby increasing the positive work of concentric phase to overcome the negative work. Countermovement is described as a movement in a direction opposite to the goal direction. Some tasks such as throwing and jumping start with countermovement in order to improve performance. Countermovement is commonly observed in actions involving SSC. The pre-stretch in countermovement leads to higher speed and greater force production in athletic performance. The spring-like mechanism found in SSC actions, ${ }^{5}$ could be applied to explain the superior results gained in actions involving countermovement. The coil of spring immediately rebounds and jumps off the surface in an opposite direction after it is released from compression. The spring jumps higher or farther by increasing the compression speed and compression force applied on the spring. This is known as the "rate of loading". Studies related to the acute and chronic effects brought by AEL were inconclusive, mostly due to differences in selecting subjects, exercise, load prescription, and method of providing AEL. ${ }^{5}$ some studies demonstrated that the AEL protocol could acutely enhance athletic performance while some studies showed the contrary results. Therefore, further research should be conducted to evaluate the training effects of AEL among untrained subjects because training the untrained individual is the initial step of novice training programs. Moreover, there is lack of study conducted to investigate the effects of AEL on standing long jump (broad jump) performance. Standing long jump is an athletic event and becomes one of the annual events 
in Norwegian Championship since 1995. In addition, it is frequently implemented in training regimen to improve athletic performance, especially lower body strength and explosive power. The authors from a study suggested trainers should use long jump training instead of vertical jump to improve swimming start performance. According to, sprinting speed in rugby could be enhanced by increasing lower body strength. Some studies have demonstrated that standing long jump has strong correlation with sprinting by sharing common physiological and biochemical determinants. ${ }^{7}$ Therefore, more studies related to improving jump performance are encouraged in order to benefit the athletes who reach plateau stage of performance and athletes who just recover from injury and wish to reach targeted performance with minimizing injury risk in a short period. The aim of this study was to evaluate the training effects of accentuated eccentric load countermovement jump on horizontal jump distance and maximum muscle strength of lower extremity among young adults.

\section{Methods}

We adopted a randomized controlled trial design to determine the effects of accentuated eccentric training. Participants had been categorized into intervention group (Accentuated Eccentric Jump) and control group (Body Mass Jump) using block randomization to ensure that roughly equal numbers of samples are randomly assigned to the two groups in a way that both known and unknown prognostic factors are balanced at the start of the trial. Randomization was performed using computer-generated random numbers. Thirty young adults (16 males, 14 females) were recruited among students of University Tunku Abdul Rahman (UTAR) Sungai Long Campus. Subjects were screened for eligibility according to the inclusion and exclusion criteria. Subjects of either gender of age between 18-23 years with Body Mass Index between 18.5 and 23 (According to Asian Standard) were included in this trial. Physically healthy subjects screened by Physical Activity Readiness Questionnaire (PAR-Q) as well as untrained subjects; not trained in a particular skill for sports were eligible to participate in this trial. Subjects with spinal problems, hypermobile, unstable and painful joints were excluded. The study was conducted at UTAR Gymnasium. Subjects were excluded if there was any history of musculoskeletal injury in the last six months, cardiovascular disease and congenital malformations. Participants were assigned randomly to two groups; either in control (BMJ) group or intervention (AEJ) group. All participants were informed about the potential risks of the experiment and written informed consent was obtained from the participants prior to the trial. The experiments were performed in accordance with the ethical standards of the Helsinki Declaration. This study was approved by the Scientific and Ethical Review Committee (SERC) of UTAR. The subjects were informed about the scope and protocol of the trial, and that they are free to withdraw from the trial at any point of time.

\section{Outcome measurements}

\section{Standing long jump test}

Standing long jump (SLJ) test is commonly used to measure the lower limb explosive power and general muscular fitness by determine horizontal jump distance. The athlete was instructed to stand behind a line marked on the ground with feet slightly apart. A two foot takeoff and landing was used, with swinging of the arms and bending of the knees to provide forward drive. The subject attempts to jump as far as possible, landing on both feet without falling backwards.
Three attempts were allowed. It is a time and cost-efficient method without requiring any high-technology equipment. Standing long jump test could be used as a tool to measure lower limb power when laboratory method is not feasible. ${ }^{8}$ conducted a study to investigate the feasibility of standing long jump test to assess the lower limb power in elite sportsmen when the Wingate test is not practical to be applied and indicated that standing long jump test is a reliable and accurate method for the assessment.

\section{5-repetition maximum strength test (5RM squat test)}

5RM Squat Test is proclaimed to be a reliable instrument to assess muscular strength in recreational sports. Subjects had preparatory session with 20 repetitions of standing back extension exercise and 20 repetitions of squat without load. Before test session, the bar was placed on the rack according to the height of subjects. Subjects were advised to wear tight weight belt to minimize the risk of injury. A prefamiliarization session without additional weight was scheduled prior to actual test. Subjects were instructed to dismount the bar from the rack, place on the back shoulder and stand in an upright position with feet shoulder-width apart. It was followed by squat until reaching approximately $110^{\circ}$ of knee flexion with greater trochanter of femur and superior border of patella were in same horizontal plane. Then, subjects ascend to original position according to verbal sign given by assessor. The load was increased with $5-10 \mathrm{~kg}$ for every trial until subjects was unable to lift the weight sixth repetition for the trial. The 5-RM strength test is a valid measurement method in recreational sports with less physical stress and injury risk. ${ }^{9}$

\section{Training protocol}

Prior to training, general warming up session was conducted by subjects, including 10 minutes of cycling, approximately five minutes of dynamic stretching (hip flexor, hip extensor, knee extensor, knee flexor and ankle plantar flexor), 10minutes of dynamic activities (three sets of one-minute skips, two minutes of leg swings, two minutes of arm swings) as well as 10 simple squats. After warming up session, three to five minutes of resting time were given to subjects before starting training session. The subjects in the accentuated eccentric training and normal strength training groups were tasked with performing a 4-week training program made up of 3 training sessions per week. A pre-familiarization session was provided for standing long jump and squatting with barbell. The subjects in AEJ group held dumbbells in each hand during eccentric phase of the jump and dropped the dumbbell before commencing the concentric phase of jump. AEJ group was advised to jump in a pattern as similar as natural countermovement jump. Moore and Schilling proposed using dumbbells and therabands to provide accentuated eccentric loading. BMJ group was encouraged to use arm swing in the propulsive phase. The numbers of session and repetition were increased every week, as outlined in Table 1.

Table I Training volume of AEJ \& BMJ groups

\begin{tabular}{lllll}
\hline Exercise & $\begin{array}{l}\text { Week I } \\
\text { S\&R }\end{array}$ & $\begin{array}{l}\text { Week } 2 \\
\text { S\&R }\end{array}$ & $\begin{array}{l}\text { Week 3 } \\
\text { S\&R }\end{array}$ & $\begin{array}{l}\text { Week 4 } \\
\text { S\&R }\end{array}$ \\
\hline AEJ & $4 \times 5$ & $6 \times 5$ & $8 \times 5$ & $10 \times 5$ \\
BMJ & $4 \times 5$ & $6 \times 5$ & $8 \times 5$ & $10 \times 5$ \\
\hline
\end{tabular}

S, Session; R, Repetition 


\section{Statistical analyses and results}

Statistical analyses were performed by using SPSS statistical package version 23. The data of horizontal jump distance collected from SLJ test and maximum muscle strength of lower extremities collected from 5RM squat test of both groups were statistically analyzed to compare the effects between AEJ and BMJ groups. The normality of collected data of SLJ test and 5RM squat test at pre-test and post-test session for both groups were statistically determined by Shapiro-Wilk Test in order to be qualified for using parametric test. Paired-Sample $t$ test was used to compare pre-test and post-test mean of SLJ test and 5RM squat test within groups. Unpaired t test was used to compare pre-test and post-test mean of SLJ test and 5RM squat test between intervention group and control group. Descriptive statistics were done in the form of mean, standard deviation (SD). Table 2 outlined that pre-test values of dependent variables were normally distributed according to Shapiro-Wilk test. The p-values for all dependent variables were more than 0.05 which showed that it was statistically insignificant for unequal distribution. Therefore, the parametric tests were suitable to analyze the data. Table 3 shows the mean difference -11.042 with standard deviation of 6.781 between pre-test and post-test $5 \mathrm{RM}$ squat test and mean difference $-\mathrm{O} .185$ with standard deviation of 0.094 between pre-test and post-test SLJ in AEJ group that was statistically significant with $\mathrm{p}-$ value of less than $(p<0.05)$. AES group demonstrated statistically significant difference in 5-Repetition Maximum Strength Test and Standing Long Jump Test.

Table 2 Determining normality of dependent variables

\begin{tabular}{llll}
\hline & Group & Mean \pm SD & P value \\
\hline \multirow{2}{*}{ Pre SLJ $(\mathrm{m})$} & BMJ & $1.529 \pm 0.312$ & 0.209 \\
& AEJ & $1.708 \pm 0.293$ & 0.851 \\
Pre 5RM $(\mathrm{kg})$ & BMJ & $43.654 \pm 14.917$ & 0.111 \\
& AEJ & $47.708 \pm 8.425$ & 0.266 \\
\hline
\end{tabular}

Table 3 Paired-samples T test of pre-test and post-test of SLJ test and 5RM in $\mathrm{AEJ}$ group

\begin{tabular}{|c|c|c|c|c|c|}
\hline $\begin{array}{l}\text { Outcome } \\
\text { measure- } \\
\text { ments }\end{array}$ & Pre test & $\begin{array}{l}\text { Post } \\
\text { test }\end{array}$ & $\begin{array}{l}\text { Mean } \\
\text { difference }\end{array}$ & $\begin{array}{l}\mathbf{T} \\
\text { value }\end{array}$ & $\begin{array}{l}P \\
\text { value }\end{array}$ \\
\hline $\mathrm{SLJ}(\mathrm{m})$ & $1.708 \pm 0.293$ & $\begin{array}{l}1.893 \pm \\
0.270\end{array}$ & $\begin{array}{l}-0.185 \pm \\
0.094\end{array}$ & $-6.823^{*}$ & 0.001 \\
\hline $5 \mathrm{RM}(\mathrm{kg})$ & $47.708 \pm 8.425$ & $\begin{array}{l}58.750 \pm \\
8.758\end{array}$ & $\begin{array}{l}-11.042 \pm \\
6.781\end{array}$ & $-5.64 I^{*}$ & 0.001 \\
\hline
\end{tabular}

*Paired-Samples T Test was performed, level of significance $\mathrm{p}<0.05$

\section{Discussion}

This study evaluated the effects of six week accentuated eccentric load countermovement jump training on horizontal jump distance and maximum muscle strength of lower extremities among young adults. The AEJ protocol was developed with the suggested potential physiological mechanisms (neural stimulation, stretching of parallel and series muscle-tendon complex, elasticity of tendon, pre-tension as well as effects of PAP) as mentioned in the introduction. This study followed the progression of training as mentioned in the study conducted by.$^{10}$ The results of the current study are in accordance with the findings reported by previous studies on Accentuated eccentric training. Accentuated eccentric training seems to be routinely superior to normal strength training when either isometric or eccentric strength are measured. The study revealed that by accentuating the eccentric phase a 'mean improvement' of $85 \%$ was gained compared to that of concentric only training of $78 \% .{ }^{11}$ Additional eccentric load promotes greater number of motor units to be recruited and the elastic properties of muscle allow for a more powerful concentric contraction after a stretch associated with the eccentric contraction. During eccentric loading the agonist muscle can achieve an active state and force as a result of some cross bridges being attached prior the subsequent portion of an exercise. ${ }^{12}$ Another study conducted to compare the strength and neuromuscular adaptations reported significantly greater increases in concentric 1RM of the elbow extensors. ${ }^{13}$ A study published in mid-2016 discovered that just five weeks of accentuated eccentric loading training provided highly noticeable results, even among experience resistance trainees. The findings of this study by a team of scientists from the Department of Biology of Physical Activity at University of Jyvaskyla in Finland indicated visible improvement in their work capacity, resistance, muscle activation, force production and muscle cross-sectional area. ${ }^{14}$ Additional eccentric load enhances eccentric impulse, the rate of force development and will result in increase in integrated electromyography across the eccentric phase. The fact that the results were so visible even among experienced strength trainees means that accelerated eccentric loading training could be a good option for anyone to get over their plateau. This assumption has been proved by the findings of the current study conducted among untrained young adults. Accelerated eccentric loading training is basically the reverse of regular training. Instead of placing emphasis on the concentric (shortening) phase of the exercise, the emphasis is on the eccentric (lengthening) phase of the exercise. The greater external load is applied during the eccentric phase. In addition, eccentric overload training showed superior results in the percentage of type IIA fibres expressing MHC IIx mRNA, in the cross-sectional area of type IIB fiber, in the level of mRNA of fast, glycolytic fibres, and in the level of capillary lactate after exercises as compared to traditional strength training. ${ }^{15}$ Post-exercise lactate clearance was found significantly greater in accentuated eccentric training compared to traditional strength training. This factor along with greater take-off speed could yield greater jump distance could have acted in favor of subjects that underwent AEJ training to enhance their athletic performance. Previous studies have reported that drop jumps result in exercise induced muscle damage, but the extent of this damage is reduced after subsequent bouts of drop jumps. ${ }^{16}$ Traditional training focuses primarily on the concentric phase of the workout. The eccentric phase is usually used to give the muscles a break between exertions, but according to the research, it could be the best time to push the muscle harder. Paying more attention to muscles during the eccentric phase of any exercise is a good way to essentially access untapped strength. ${ }^{17,18}$

\section{Conclusion}

Implementing Accentuated Eccentric Loading training to strengthening has shown to bring out gains in horizontal jump distance and maximum muscle strength of lower extremity among young adults. This is a fantastic nugget of knowledge for all athletes. In conclusion, this study has shown that jump performance and muscle strength can be enhanced by accentuated eccentric muscle action when compared with a traditional protocol. This may provide a more practical method for coaches to enhance short-term explosive movements in athletic populations. 


\section{Acknowledgements}

The authors thank all the participants and the staff of UTAR gymnasium. This study was not funded by any person or agency. The authors would like to acknowledge Ajmal Sheriff, Sport Physiotherapist for his continuous encouragement and supervision in implementing knowledge on this subject.

\section{Conflict of Interest}

Author declares that there is no conflict of interest.

\section{References}

1. Miyama M, Nosaka, K. Protection against muscle damage following fifty drop jumps conferred by ten drop jumps. J Strength Cond Res. 2007;21(4):1087-1092.

2. Newton RU, Kraemer WJ, Hakkinen K. Effects of ballistic training on preseason preparation of elite volleyball players. Med Sci Sports Exerc. 1999;31:323-330.

3. Barr MJ, Sheppard JM, Agar-Newman DJ, et al. Transfer effect of Strength and Power Training to the Sprinting Kinematics of International Rug by Players. J Strength Cond Res. 2014;28(9):2585-2596.

4. Krishnan A, Sharma D, Bhatt M, et al. Comparison between Standing Broad Jump test and Wingate test for assessing lower limb anaerobic power in elite sportsmen. Med J Armed Forces India. 2017;73(2):140 145

5. Wilson A, Lichtwark G. The anatomical arrangement of muscle and tendon enhances limb versatility and locomotor performance. Philos Trans Royal Soc B. 2011;366(1570):1540-1553.

6. Gail S, Kunzell S. Reliability of a 5-Repetition Maximum Strength Test in Recreational Athletes. Dtsch Z Sportmed. 2014;11:314-317.

7. Peloquin A. How Accentuated Eccentric Loading Leads to Greater Strength Gains. USA; 2016.

8. Wagle JP, Taber CB, Cunanan AJ, et al. Accentuated Eccentric Loading for Training and Performance: A Review. Sports Med. 2017;47(12):24732495.
9. Sheppard JM, Young K. Using additional eccentric loads to increase concentric performance in the bench throw. J Strength Cond Res. 2010;(10):2853-2856

10. Sheppard J, Hobson S, Barker M, et al. The Effect of Training with Accentuated Eccentric Load Counter-Movement Jumps on Strength and Power Characteristics of High-Performance Volleyball Players. Int J Sports Sci Coach. 2008;3(3):355-363.

11. Hortobagyi T, De Vita P. Favorable neuromuscular and cardiovascular responses to 7 days of exercise with an eccentric overload in elderly women. J Gerontol A Biol Sci Med Sci. 2000;55(8):401-410.

12. Friedmann-Bette B, Bauer T, Kinscherf R, et al. Effects of strength training with eccentric overload on muscle adaptation in male athletes. Eur J Appl Physiol. 2010;108(4):821-836.

13. Brandenburg JP, Docherty D. The effects of accentuated eccentric loading on strength, muscle hypertrophy, and neural adaptations in trained individuals. J Strength Cond Res. 2002;16(1):25-32.

14. Walker S, Blazevich AJ, Haff GG, et al. Greater Strength Gains after Training with Accentuated Eccentric than Traditional Isoinertial Loads in Already Strength-Trained Men. Front Physiol. 2016;27:7-149.

15. Vogt M, Hoppeler HH. Eccentric exercise: mechanisms and effects when used as training regime or training adjunct. $J$ Appl Physiol. 2014;116(11):1446-1454.

16. Myer GD, Ford KR, Brent JL, et al. Predictors of sprint start speed: The effects of resistive ground-based vs. inclined treadmill training. $J$ Strength Cond Res. 2007;21:831-836.

17. Rebutini VZ, Pereira G, Bohrer RCD, et al. Plyometric Long Jump Training With Progressive Loading Improves Kinetic and Kinematic Swimming Start Parameters. J Strength Cond Res. 2016;30(9):23922398.

18. Asadi A. Relationship between Jumping Ability, Agility and Sprint Performance of Elite Young Basketball Players: A Field-Test Approach. Rev Bras Cineantropom Desempenho Hum. 2016;18(2):177-186. 